\title{
Tau Isoform with Three Microtubule Binding Domains is a Marker of New Axons Generated from the Subgranular Zone in the Hippocampal Dentate Gyrus: Implications for Alzheimer's Disease
}

\author{
María Llorens-Martin ${ }^{\mathrm{a}}$, Cátia M. Teixeiraa ${ }^{\mathrm{c}, \mathrm{d}}$, Almudena Fuster-Matanzo ${ }^{\mathrm{a}}$, Jerónimo Jurado-Arjona ${ }^{\mathrm{a}}$, \\ Víctor Borrelle ${ }^{\mathrm{e}}$ Eduardo Soriano ${ }^{\mathrm{b}, \mathrm{c}, \mathrm{d}}$, Jesús Avila $^{\mathrm{a}, \mathrm{b}}$ and Félix Hernández ${ }^{\mathrm{a}, \mathrm{b}, *}$ \\ ${ }^{a}$ Centro de Biología Molecular Severo Ochoa, CSIC/UAM, Madrid, Spain \\ ${ }^{\mathrm{b}}$ CIBERNED, Centro de Investigación Biomédica en Red de Enfermedades Neurodegenerativas, Madrid, Spain \\ ${ }^{\mathrm{c}}$ Developmental Neurobiology and Regeneration Laboratory, Institute for Research in Biomedicine, Barcelona, \\ Spain \\ ${ }^{\mathrm{d}}$ Department of Cell Biology, University of Barcelona, Barcelona, Spain \\ ${ }^{\mathrm{e}}$ Developmental Neurobiology Unit, Instituto de Neurociencias. CSIC/Universidad Miguel Hernández, Sant Joan \\ d'Alacant, Alicante, Spain
}

Handling Associate Editor: Khalid Iqbal

Accepted 17 January 2012

\begin{abstract}
In the adult hippocampal dentate gyrus, newborn granule cells grow dendrites into the molecular layer and send axons into the CA3 region. Several molecular markers have been used to analyze production of these new neurons; however, no good markers for new axons have been described. Here we demonstrate that tau protein isoform with three microtubule binding domains (3R-Tau) is a marker of those axons following an antigen retrieval protocol. By using retrovirus-mediated GFP transduction, GFP can be detected in a period of 7-14 days after viral infection. We also provide a "proof of principle" demonstration of the power of that labeling showing modulation of 3R-Tau positive axons under physiological conditions (exercise and aging) as well as in a FTDP-17 neurodegenerative model and Alzheimer's disease models (mice overexpressing A $\beta P P s w$, ind or GSK3 $\beta$ ). We conclude that $3 R$-Tau would be an efficient marker and a valuable tool to study new axons in adult neurogenesis as well as in neurodegenerative processes.
\end{abstract}

Keywords: Adult neurogenesis, dentate gyrus, subgranular zone, tau protein

\footnotetext{
*Correspondence to: Félix Hernández, Centro de Biología Molecular Severo Ochoa, CSIC/UAM, Madrid, Universidad Autónoma de Madrid, Campus de Cantoblanco, 28049 Madrid, Spain. Tel.: +34 911964563; Fax: +34 911964420; E-mail: fhernandez@cbm.uam.es.
}

\section{INTRODUCTION}

The subgranular zone (SGZ) of the hippocampal formation is a region where neurogenesis takes place in adult mammals. In the hippocampus, newly differentiated granule cells grow dendrites into the molecular 
layer and send axons into the CA3 region. Major glutamatergic synaptic activation from perforant path afferents does not occur until new neurons are two or more weeks old. It coincides with appearance of spines on dendrites of newly born neurons [1]. Within four to six weeks after birth, these neurons become fully integrated in the circuit [2]. The stem cells that exist are a subset of astrocytes [3]. In the SGZ, astrocytes-like (Type B cells) divide to generate intermediate precursors (Type D cells), which remain in clusters of two to four cells in neurogenic niches that are formed by the processes of astrocytes and specialized vasculature [4]. Type D cells progressively generate more differentiated progeny, which eventually mature into granule neurons. There are good markers for labeling different maturative stages of the cellular progeny, but these markers usually label nuclei, soma, or somatodendritic compartments. However, no good markers for new-generated axons have been described. Axons from granule neurons form mossy fibers in the hilar region of dentate gyrus (DG) can be visualized after zinc precipitation using Timm staining. In addition, immunolabeling of zinc transporter-3 which labels synaptic vesicles of granule cell mossy fibers or the calcium binding protein calbindin have been used. However, these approaches label whole perforant tract formed by mature granule cell axons, but no individualized axons can be observed with these two methods. To visualize new axons, fluorescent retrograde tracers [5] and retroviral expression of green florescent protein have been used [6], but inflammation, degeneration, or DG structure alteration may be an important technical disadvantage that should be taken into account. Nevertheless, if we adopt the theory of recapitulation or embryological parallelism to adult neurogenesis, it can be accepted that adult neurogenesis recapitulates neuronal development [7]. Thus, neuronal cytoskeleton offers us some specific markers which participate in the process of morphological and physiological maturation that takes place in these cells after commitment to neuronal lineage. One of these proteins is tau protein.

Tau is a neuronal microtubule-associated protein that stabilizes neuronal microtubules under normal physiological conditions. Tau plays a key role in the morphogenesis of neurons. The human tau gene contains 16 exons from which different tau isoforms are generated by alternative splicing $[8,9]$. Some of these isoforms are selectively expressed during embryonic and early postnatal development [9-11], whereas in the human adult central nervous system, six different tau isoforms are expressed that differ in the presence or absence of exons 2, 3, and 10 [12]. Exon 10 encodes one of the four repeat sequences $[9,12]$ that form the microtubule-binding domain [13]. The presence of exon 10 results in tau with four repeat microtubulebinding sequences (4R), whereas the alternatively spliced isoforms without exon 10 have only three of these sequences (3R). The expression of some of these tau isoforms is developmentally regulated. Thus, isoforms lacking exon 10 (3R-Tau) are found at early developmental stages whereas tau isoforms containing exon 10 (Tau4R) are mainly found in neurons at mature developmental stages [14].

In this paper, we demonstrate that tau protein isoform with three microtubule binding domains (3R-Tau) is a marker of new axons generated from the SGZ. In addition, we also provide a "proof of principle" demonstration of the power of that labeling showing modulation of 3R-Tau positive axons under physiological (exercise and aging) and pathological conditions (in FTDP-17 and Alzheimer's disease (AD) neurodegenerative mouse models).

\section{MATERIALS AND METHODS}

\section{Animals}

Eight week old wild-type (wt) male (C57BL/6J) mice were bred at Centro de Biología Molecular Severo Ochoa (Madrid, Spain). Animals were housed at $22 \pm 1^{\circ} \mathrm{C}$ on a $12 / 12 \mathrm{~h}$ light/dark cycle, with ad libitum access to food and water. Mice were kept under standard laboratory conditions in accordance with European Community Guidelines (directive 86/609/EEC). All animals were handled in strict accordance with good animal practice as defined by the national animal welfare bodies and institutional guidelines. The following animal models have been used: young exercised mice and age-matched sedentary control mice as described previously [15]; a murine model of Frontotemporal Dementia linked to Parkinsonism associated to chromosome 17 (FTDP-17) (TauVLW mice) [16]; Glycogen Synthase Kinase $3 \beta$-overexpressing mice (GSK3 $\beta$-OE mice) [17], and murine model overexpressing A $\beta$ PPind, sw (A $\beta P P$ with Indiana and Swedish mutations) [18].

\section{Sacrifice}

Mice were completely anaesthetized with an intraperitoneal (IP) pentobarbital injection. Animals were then perfused with saline followed by $4 \%$ paraformaldehyde in phosphate buffer. The brains of 
the mice were removed and post-fixed overnight in the same fixative.

\section{Histology}

Sagittal sections $(50 \mu \mathrm{m}$ thick) from one hemisphere were obtained on a Leica VT1000S vibratome and series of sections were generated comprised of every 8 th section. Double or triple immunohistochemistry was performed as described previously [15]. For 3R-Tau detection, sections were processed for antigen retrieval. Sections were immersed in antigen retrieval solution, Citra Plus (BioGenex, Cat.Nos. HK080-9K) and microwave irradiated following manufacturer protocol. Then, a standard protocol for immunohistochemistry was carried out, incubating the sections with the following primary antibodies for 24 to $48 \mathrm{~h}$ : mouse anti-3R-Tau (1 : 500, Millipore Cat. Number: 05-803); rabbit antiCalbindin $(1: 1000$, Swant); goat anti-doublecortin (DCX, 1:500, Santa Cruz); rabbit anti-calretinin (1:2000, Swant). The binding of these antibodies was then detected over $24 \mathrm{~h}$ at $4^{\circ} \mathrm{C}$ with the following donkey Alexa-conjugated secondary antibodies as appropriate $(1: 1000$, Molecular Probes, Eugene, OR, US): Anti-rabbit Alexa 555-conjugated (Calbindin and calretinin detection); Anti-goat Alexa 633-conjugated (Doublecortin detection); and Antimouse Alexa 488-conjugated (3R-Tau detection). All the sections were counterstained for $10 \mathrm{~min}$ with DAPI (1:1000, Calbiochem-EMD Darmstadt, Germany).

For amyloid- $\beta$ antigen retrieval, a 10-min formic acid $(70 \%)$ incubation was performed prior to microwave irradiation. A rabbit anti-human amyloid- $\beta$ (Millipore; $1: 1000$ ) was used as a primary antibody followed by incubation with the appropriate secondary antibody as previously described.

Sections were observed under a Zeiss confocal microscope (Zeiss LSM710). Mosaics of low magnification images were obtained using a 10X immersion oil objective and composed using the Zeiss software "Title Scan" tool.

\section{Retrovirus injection}

CAG-GFP retroviruses encoding for gfp [1] were used. Plasmids used for the production of GFP expressing retrovirus were a generous gift of Fred H. Gage. Retroviruses were produced by transfection of the 293 cell line as described previously [1]. Retroviral stocks were ultracentrifugated and concentrated to working titers of $1 \times 10^{7}-2 \times$ $10^{8} \mathrm{pfu} / \mathrm{ml}$.

Mice were pre-anaesthetized by IP atropine sulfate $(0.1 \mathrm{mg} / \mathrm{kg})$ injection, then anesthetized with chloral hydrate $(400 \mathrm{mg} / \mathrm{kg}$, IP), and given $5 \mathrm{mg} / \mathrm{kg}$ of ketoprofen subcutaneously and placed in a stereotaxic frame. The scalp was incised and skull hole(s) drilled. Coordinates $(\mathrm{mm})$ relative to bregma in the anteroposterior, mediolateral, and dorsoventral planes were as follows: DG $[-2.0,1.4,2.2] .1 .5 \mu \mathrm{l} / \mathrm{DG}$ of virus solution was infused at $0.2 \mu \mathrm{l} / \mathrm{min}$ via glass micropipette, leaving the micropipettes in place an additional $5 \mathrm{~min}$ to ensure diffusion.

\section{RESULTS}

\section{Axonal labeling with 3R-Tau antibody label DCX-positive cells}

We first tested if 3R-Tau antibody was able to label new axons in the hilar portion of the DG. When we performed a standard immunolabeling protocol, the antibody anti-3R-Tau gave a slight signal in the somatic compartment (data not shown). Then, sections were processed for antigen retrieval. Thus, sections were immersed in antigen retrieval solution Citra Plus (BioGenex, Cat.Nos. HK080-9K), microwave irradiated, and then incubated for $15 \mathrm{~min}$ at $80^{\circ} \mathrm{C}$. In these new conditions, 3R-Tau antibody labeled the somatic compartment of a subpopulation of cells in the SGZ of the hippocampal DG as well as axonal processes in the hilar region and hippocampal CA3 subfield (Fig. 1A-F). To identify those cells and taking into account that $\mathrm{SGZ}$ is an area where neurogenesis takes place in adult animals and that it has been previously reported that somatodendritic tau colocalizes with DCX [19-21], we performed double immunostaining with both antibodies. Figure $1 \mathrm{E}, \mathrm{F}$ shows a panoramic view of the DG showing that in the majority of the labeled cells DCX and 3R-Tau colocalize (white asterisks) and only a few of them are single DCXpositive labeled (Fig. 1 E, F, white arrowheads). These cells probably represent those cells which initiate a neuronal program but are in the initial stage of neuronal maturation. Next we wanted to demonstrate that axonal processes observed in Fig. 1 corresponded to newborn neurons. In Fig. 1C, D, it can be observed how newborn neurons axonal tract form a bundle (white arrowheads) across the hilus travelling towards CA3 hippocampal subfield, following a maturational program similar to that observed in more mature granule 
neurons, as previously described [1]. Figure 2A-C shows another panoramic view demonstrating that $3 \mathrm{R}$ Tau-positive axons are arranged longitudinally and parallel to one another in the hilus. Figure 2D-F proves that these axons belong to DCX-positive cells present in SGZ.

\section{Retrovirus-mediated GFP transduction to study the time-course expression of $3 R$-Tau protein in new neurons}

Retrovirus-mediated GFP transduction was used to study the time-course expression of 3R-Tau protein in new neurons. Viruses were delivered to the DG of the mouse hippocampus through stereotaxic surgery. Then, mice were killed at different time points $(1,2,3$, and 8 weeks after viral injection) and GFP and 3R-Tau expression were analyzed by immunohistochemistry (Fig. 3). Axonal processes were rarely observed 1 week after viral injection, and axonal 3R-Tau immunolabeling was absent in these cells; however, a slight somatodendritic staining was observed in some of the cells analyzed (Fig. 3A-C). Two week-old neurons showed apical dendrites and axons in the hilar region. These axons, as well as the somatic compartment, were labeled with 3R-Tau antibody (Fig. 3D-I). Three week-old neurons had more elaborate dendritic arborization. In cells of this age, 3R-Tau was found in the somatic compartment of most of the cells (Fig. 3M-R), but only few of them expressed 3R-Tau in the axons (white asterisks represent 3R-Tau-positive axons whereas white arrowheads label 3R-Tau-negative ${ }^{-}$axons). This 3R-Tau staining was detectable both in the subgranular layer where newborn neuron axons begin, as well as in the hilar region and CA3 field (Fig. 3J-L). Finally, 8 weekold neurons expressed neither axonal nor somatic 3R-Tau.

\section{Modulation of 3R-Tau labeling under physiological conditions and diverse neurodegenerative models}

In the dentate gyrus of a 2 month-old mouse, abundant $3 \mathrm{R}-\mathrm{Tau}^{+}$cells can be observed, and immature axons form and organized bundles of parallel fibers can be observed through the hilus. Similar to mature granule neuron axons, immature axonal fibers are organized and directed toward the CA3 hippocampal region (Fig. 4A-E). Young exercised mice show higher number of immature neurons (Fig. 4F, H, I) than agematched sedentary control mice [15], as well as higher numbers of immature axons (Fig. 4J). During aging, a decrease in the number of immature neurons has been reported [22]. In Fig. $4 \mathrm{~N}$ and $\mathrm{O}$, a reduction in the number of immature axons can be observed, related to the decrease in the number of immature neuroblasts. However, in Fig. 4O, it can be observed how organization of immature axons remains unaltered compared to that of young wt animals. A common feature of several neurodegenerative diseases is the alteration of adult hippocampal neurogenesis rate [22]. In a murine model of FTDP-17 (TauVLW mice), in which mutated human tau expression is known to be related to a dramatic reduction in the number of immature neuroblasts [23], immature axons appear slightly disorganized in the hilar region and a reduced number of axons can be appreciated in this region (Fig. 4T). Nevertheless, in GSK3 $\beta$-OE mice, the number of immature neuroblasts is known to be drastically increased compared to that of age-matched wt mice [24], as can be observed in Fig. 4U, W, and X. Accordingly, an increased density of axonal processes can be appreciated in the hilus of this animal model (Fig. 4Y), correlating with the increase in the number of immature neuroblasts. On the contrary, in aged A $\beta$ PPind, sw mice, an AD model, abundant amyloid- $\beta$ plaques can be observed in the hippocampus and cortex of these mice. As can be seen in Fig. $4 \mathrm{Z}, \mathrm{AA}, \mathrm{AB}, \mathrm{AC}$, and $\mathrm{AD}$, neither immature neuroblasts nor immature axons can be observed in this animal model surrounding amyloid- $\beta$ plaques (Fig. 4 $\mathrm{AD})$.

\section{DISCUSSION}

Here we describe a non-invasive and reproducible labeling protocol to determine properties of new axons generated in the SGZ based in 3R-Tau isoform. The majority of the labeled 3R-Tau cells colocalize with DCX and only a few of them are single DCX-positive labeled. These cells probably represent those cells which initiate a neuronal program but are in the initial stage of neuronal maturation. Using retrovirusmediated GFP transduction, our study demonstrates that 3R-Tau antibody labels newborn neuron axonal processes and, second, that 3R-Tau protein can be detected in the axonal compartment during the second and third week of age, when axons are growing to reach the CA3 region. Finally, 8 week-old neurons expressed neither axonal nor somatic 3R-Tau.

We report here a valuable tool to analyze the rate modulation of adult dentate gyrus under physiological and pathological conditions. Several molecular 

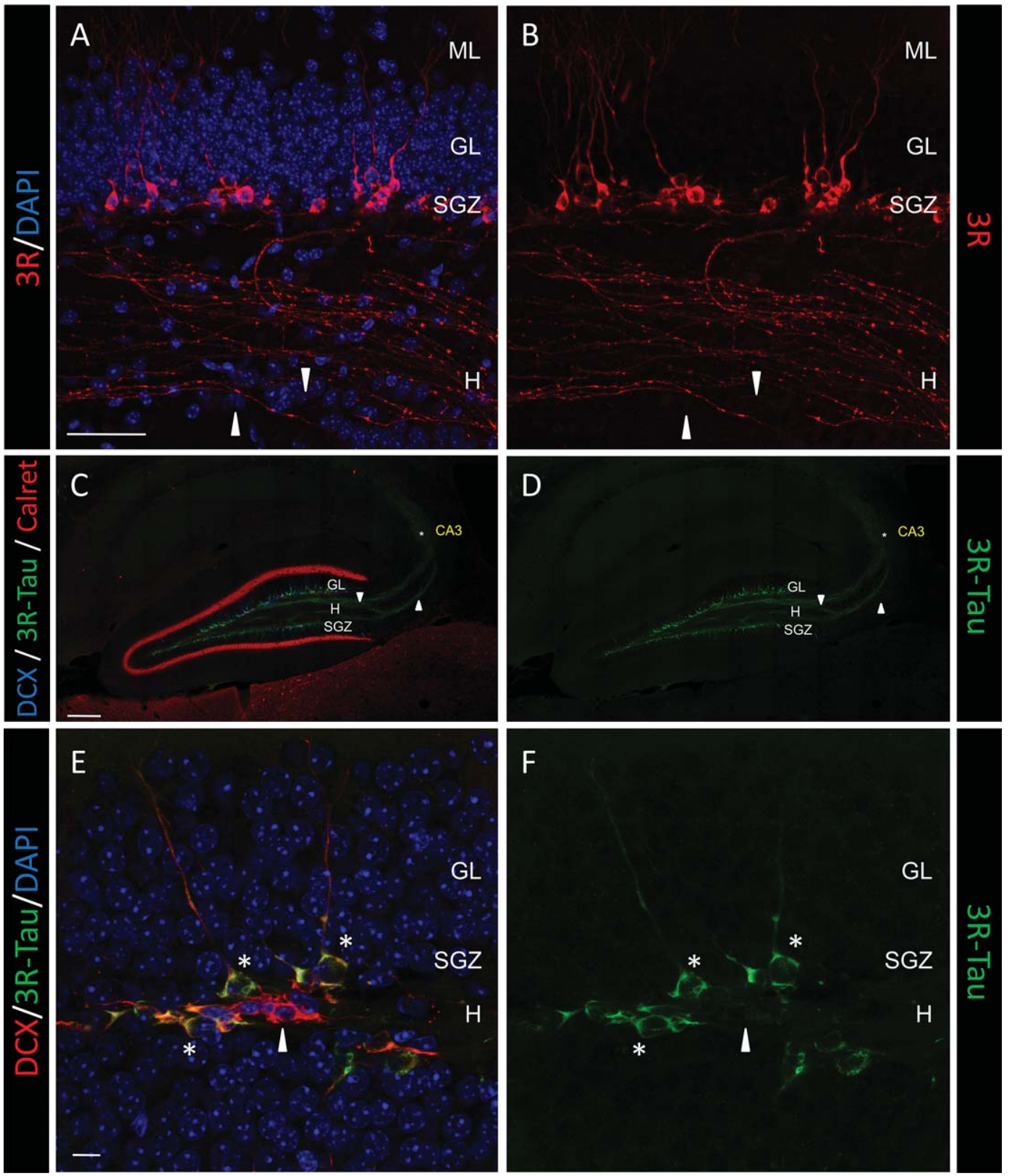

Fig. 1. 3R-Tau expressing cells in the DG. A, B) 3R-Tau (red) immunohistochemistry in 2-month-old wild-type (C57BL/6) mice showing the abundance of 3R-Tau expressing cells along the SGZ. White arrows indicate axonal processes. DAPI staining in blue. Scale bar: $50 \mu \mathrm{m}$. C, D) Triple-immunolabeling for 3R-Tau (green), DCX (blue) and calretinin (a prominent immunoreactive band at the dentate supragranular zone in red). White arrows indicate axonal processes. Scale bar: $100 \mu \mathrm{m}$. E, F) Detail of the SGZ. DCX+/3R-Tau+ (asterisks) and DCX+/3R-Tau- cells (arrowhead) can be identified. DAPI staining in blue. Scale bar: $10 \mu \mathrm{m}$. H, hilus; GL, granular layer; SGZ, subgranular layer. B, D, and F show 3R-Tau immunolabeling.

markers have been used to analyze production of new neurons in adult brain; however, no markers for new axons have been described. Thus, most of the actual investigations in adult neurogenesis only focus their studies in the somatodendritic compartment, while the axonal one has not been extensively analyzed because the lack of appropriate immunological tools. We also provide a "proof of principle" demonstration of the power of that labeling showing modulation of 3R-Tau positive axons under physiological conditions (exercise and aging) and diverse neurodegenerative (FTDP-17 and AD) models. 

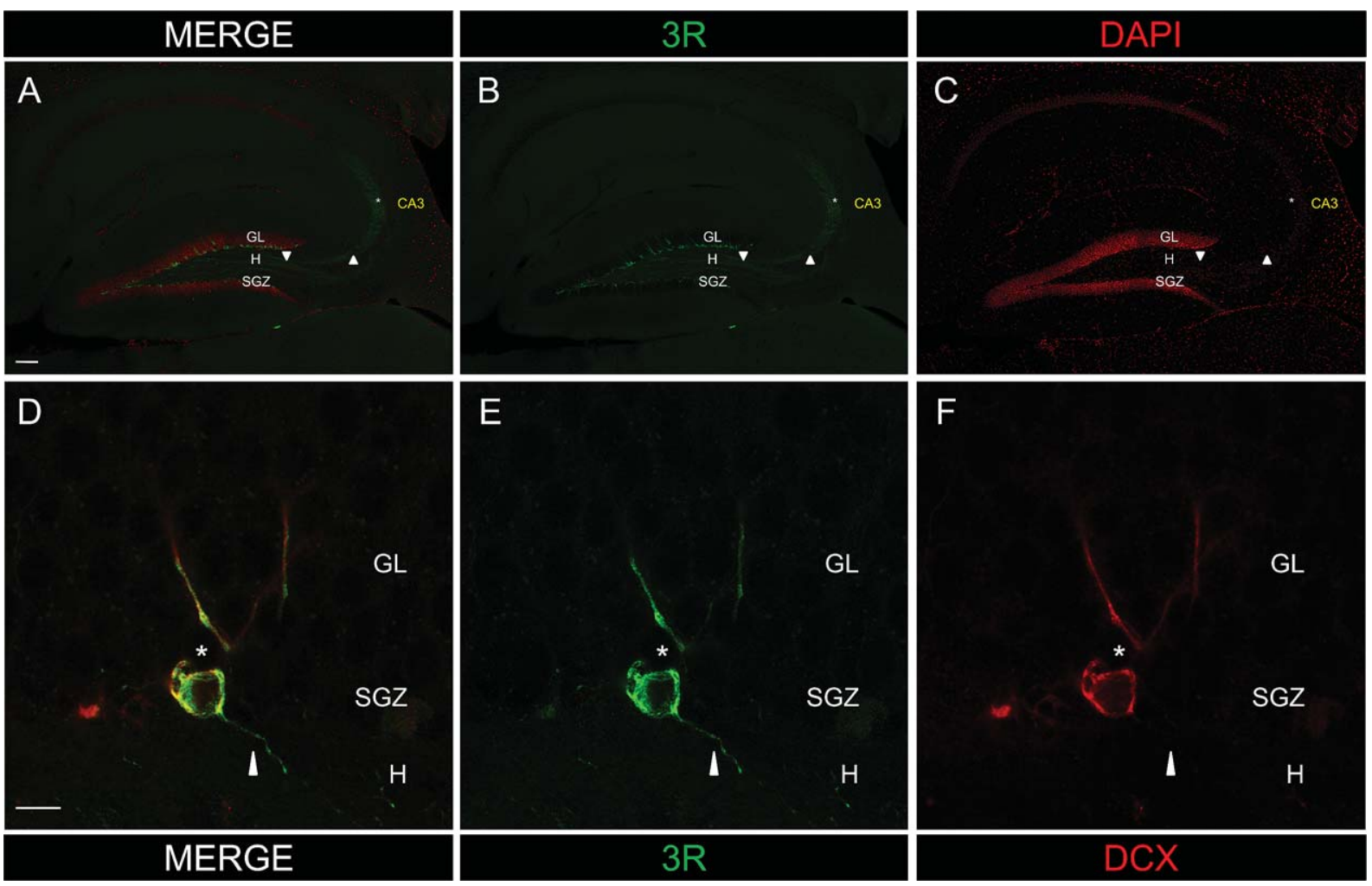

Fig. 2. 3R-Tau label axonal processes. A-C) Double immunolabeling for 3R-Tau (B, green) counterstained with DAPI (C, red). Arrows show axons arranged longitudinally and parallel to one another in the hilus. Scale bar: $50 \mu \mathrm{m}$. D-F) Magnification of a double-labeled cell showing a 3R-Tau axonal processes (arrow) rising from a DCX+ cell. Arrowhead shows axonal process emerging from a $\mathrm{DCX}^{+}$cell (white asterisk). GL, granule cell layer; $\mathrm{H}$, hilus; SGZ, subgranular zone. Scale bar: $10 \mu \mathrm{m}$.

Adult hippocampal neurogenesis is influenced both by external stimuli, such as physical exercise, and by intrinsic conditions like age and disease. We here demonstrate that new axons associated with adult neurogenesis are organized bundle of parallel fibers that can be observed through the hilus and directed toward the CA3 hippocampal region. More interestingly, young exercised mice show higher number of immature axons similar to the increase observed in the number of differentiating neurons in the granule cell layer of the mouse hippocampus [15]. Another model where a modulation of adult neurogenesis is observed is aging. During aging, a decrease in the number of immature neurons has been reported [22] and, in good agreement, a reduction in the number of immature axons labeled with 3R-Tau antibody can be observed. However, in Fig. 4O, it can be observed how organization of immature axons remains unaltered compared to that of young wt animals. Thus, 3R-Tau labeling allows not only quantitative studies of new axons, but also qualitative studies related to the degree of disorganization of these axons.

A common feature of several neurodegenerative diseases is the alteration of adult hippocampal neurogenesis rate [22]. Thus, we here reported that in a murine model of FTDP-17, in which mutated human

Fig. 3. Development of 3R-Tau labeling in newborn neurons. After retrovirus-mediated GFP transduction, mice were killed at different time points and saggital sections were immunolabeled with anti-GFP (green) and anti-3R-Tau (red). DAPI staining in blue. Arrowheads indicate GFP-expressing axons that are 3R-Tau ${ }^{-}$. Asterisks indicate GFP-expressing axons that are 3R-Tau ${ }^{+}$. A-C) 1 week post-infection. It should be noted that GFP-expressing cells send short axonal processes to hilar region. These axonal processes are $3 \mathrm{R}-\mathrm{Tau}{ }^{-}$. D-I) 2 weeks post-infection. Most of the two-week-old cells are 3R-Tau+ both in the somatodendritic area and in the axonal processes. Cells within this age send an axonal process that crosses the hilus and make its way to CA 3 region. J-R) 3 weeks post-infection. Only some of the 3 week-old cells express $3 R-$ Tau in their axons (cells represented in Fig. 3M-O), whereas other cells are 3R-Tau ${ }^{-}$(see Fig. 3P-R). At three weeks of age, most axons derived from newborn cells reach CA3 region. Fig. 3J-L shows that some of those axons are 3R-Tau ${ }^{+}$(white asterisks). S-U) 8 weeks post-infection. At this age, none of the cells expressed 3R-Tau isoform. GL, granule cell layer; H, hilus. Scale bar: $10 \mu \mathrm{m}$. 

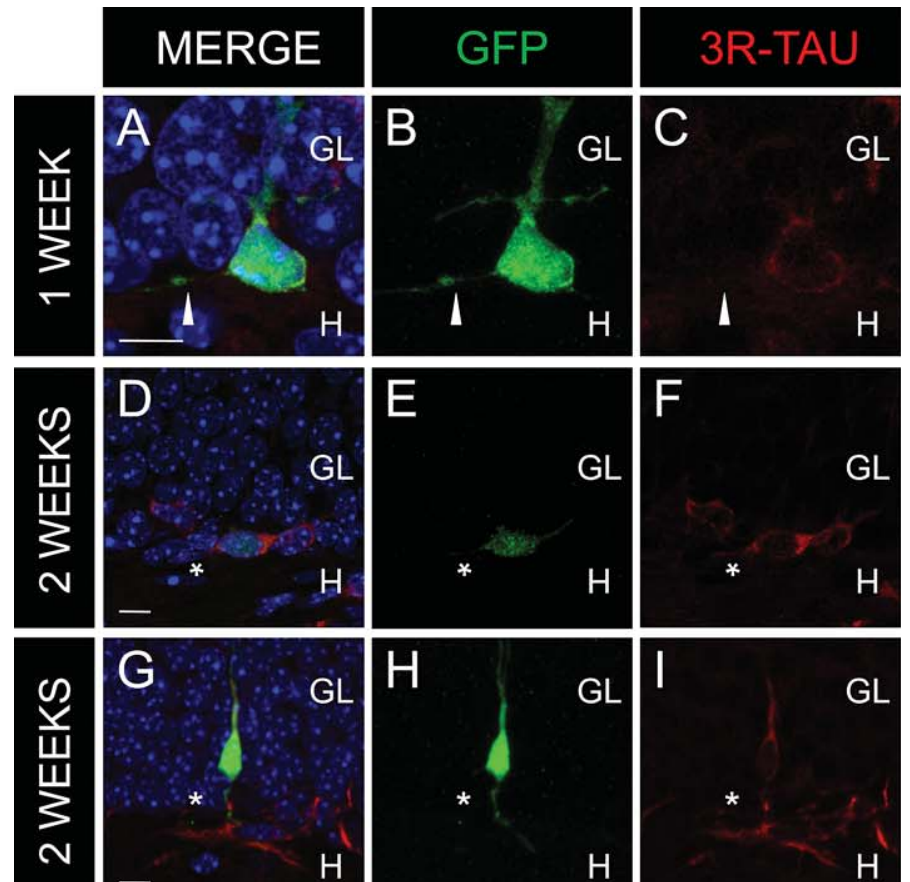

GL
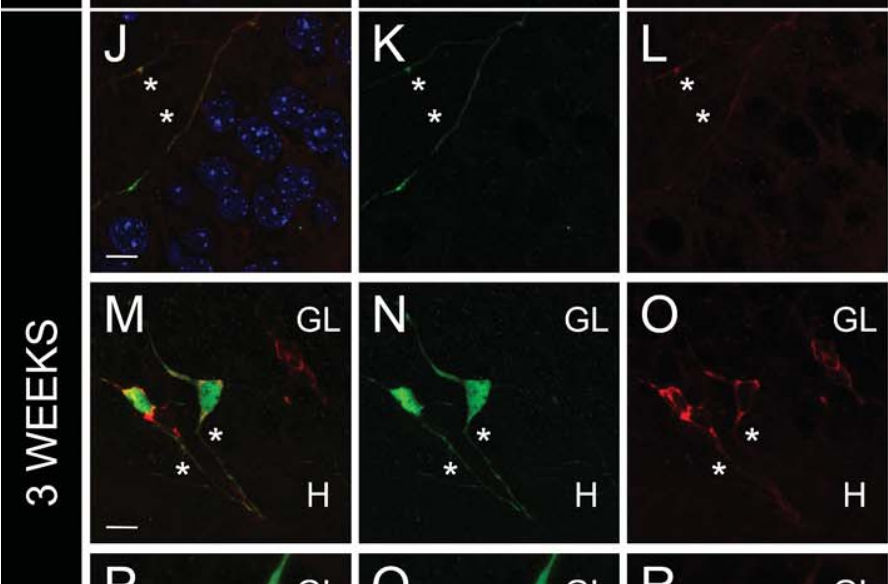

GL
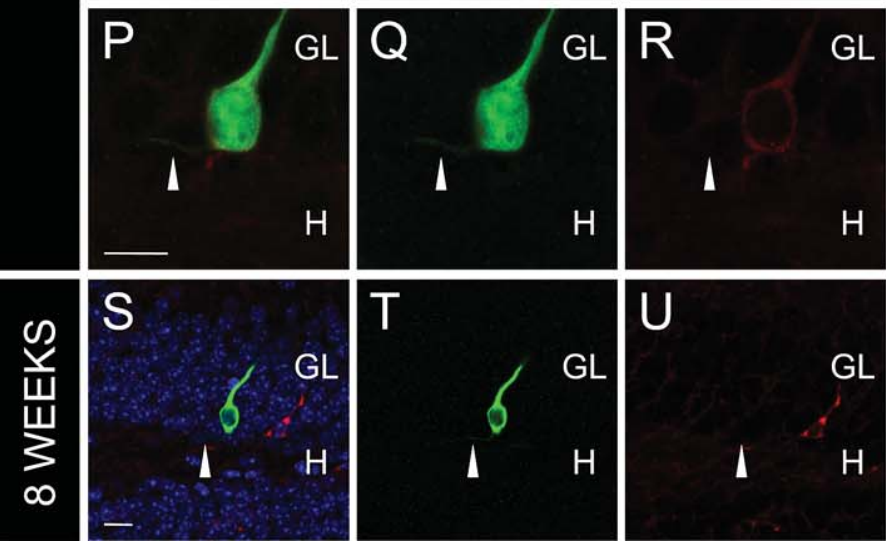

Fig. 3. (Continued) 


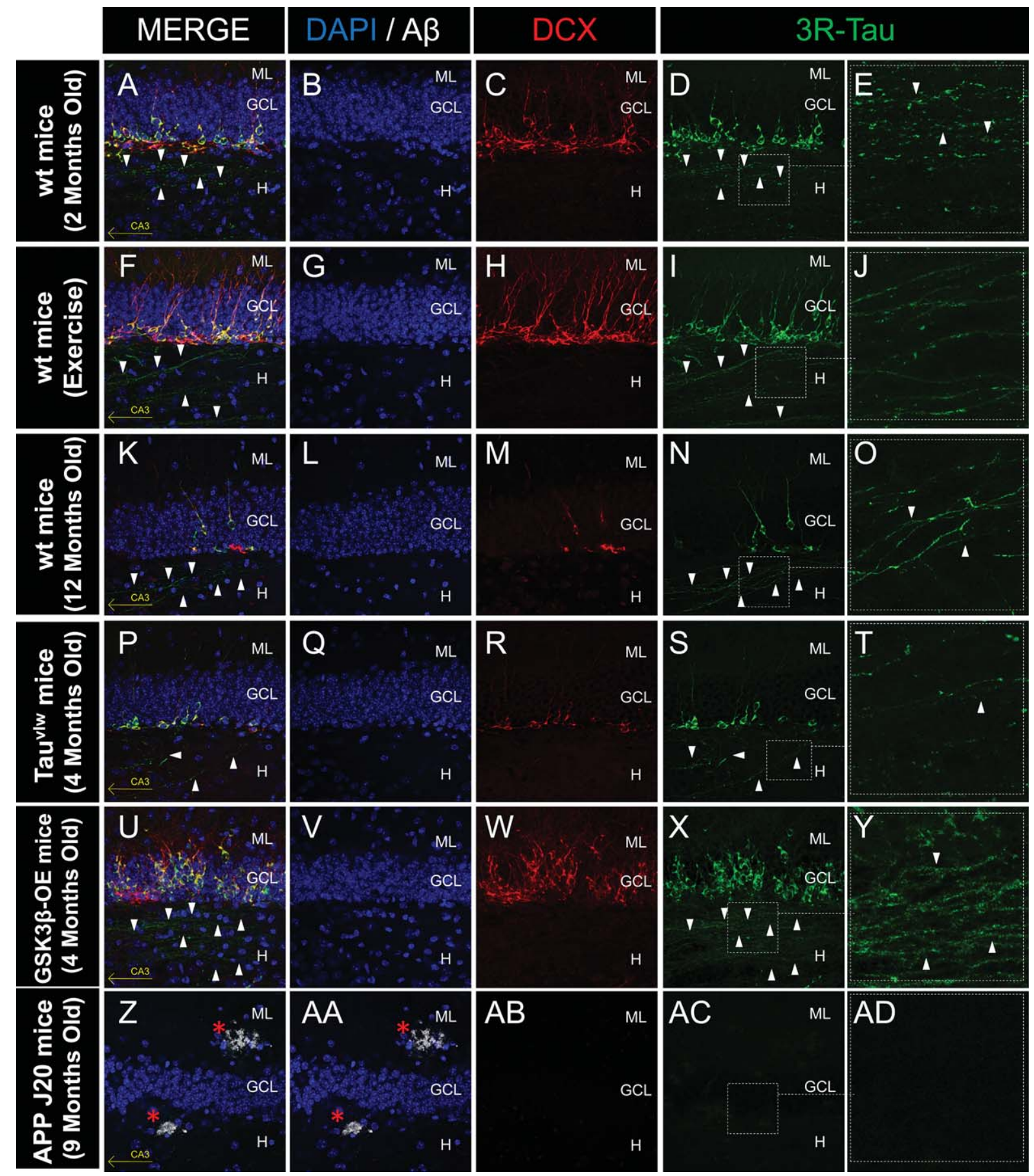

Fig. 4. (Continued) 
tau expression is known to be related to a dramatic reduction in the number of immature neuroblasts [23], immature axons appear slightly disorganized in the hilar region and a reduced number of axons can be appreciated in this region. Nevertheless, in GSK3 $\beta$-overexpressing mice, the number of immature neuroblasts is known to be drastically increased compared to that of age-matched wt mice [24]. Accordingly, an increased density of axonal processes can be appreciated in the hilus of this animal model, correlating with the increase in the number of immature neuroblasts. On the contrary, aged A $\beta P P i n d$, sw mice with abundant hippocampal amyloid- $\beta$ plaques do not show immature neuroblasts nor can immature axons be detected.

Thus, modulation of 3R-Tau positive axons under physiological conditions as well as in neurodegenerative transgenic models demonstrates that these findings have important implications in defining and understanding the role of adult neurogenesis not only in the somatodendritic compartment but also in the axonal one. 3R-Tau immunolabeling will allow the unraveling of the fundamental properties of these newborn adult axons.

New neurons require a high degree of plasticity. Tau is able to promote polymerization of tubulins [25] and prevent their dynamic instability by its binding to microtubules [26]. The 4R-Tau isoforms promote microtubule assembly at a faster rate than the 3R-Tau isoforms [27], suggesting that 3R-Tau protein may aid brain development by decreasing microtubule stability. This idea is supported by the expression of tau protein during brain development. Thus, 4R-Tau is not present in fetal human brain in contrast to adult human brain $[9,27,28]$. The same has been observed in fetal mouse brain [29, 30]. Interestingly some differences among species have been observed in adult brain in the expression of tau isoforms. Thus, 3R-Tau isoforms are not present in mature neurons of adult rodents [31,32], while the adult human brain contains both tau isoforms [14].

In conclusion, the observations summarized here favor the suggestion that tau provides a dynamic microtubule network in DCX-positive cells which allow a proper axonal growing in adult neurogenesis. In addition, and taking into account that currently no good axonal marker to study adult neurogenesis exists, we here demonstrate that 3R-Tau can be used to analyze new axons in the SGZ easily after microwave antigen retrieval. Compared to previous approaches, mainly retroviral infection, 3R-Tau immunostaining allows the rapid identification of new axons. Modulation of 3R-Tau positive axons under physiological conditions as well as in neurodegenerative transgenic models demonstrates that these findings have important implications in defining and understanding the role of adult neurogenesis not only in the somatodendritic compartment but also in the axonal one.

\section{ACKNOWLEDGMENTS}

M.L-M. is recipient of a contract from JAEDoc Postdoctoral program. We thank Elena Langa for technical support. Plasmids used for the production of GFP expressing retrovirus were a generous gift of Fred $\mathrm{H}$. Gage (Salk Institute, La Jolla, CA). This study was funded by grants from the Spanish Ministry of Health (SAF 2006-02424; SAF2010-15525), Comunidad de Madrid (SAL/0202/2006), Fundación CIEN (PI 00809), Fundación M. Botín and an institutional grant from Fundación R. Areces.

Fig. 4. Modulation of 3R-Tau under physiological conditions and diverse neurodegenerative models. A common feature of several neurodegenerative diseases is the alteration of adult hippocampal neurogenesis rate. A-D) In the dentate gyrus of a 2 month-old mouse, abundant $3 \mathrm{R}-$ Tau ${ }^{+}$ cells can be observed. E) High magnification image of squared area in D. An organized bundle of parallel fibers corresponding to immature axons can be observed through the hilus. It should be noted that immature axons are organized and directed toward the CA3 hippocampal region (left). F-J) Exercised mice show higher number of immature neurons (F, H, I) than age-matched sedentary control mice (A-D), as well as higher number of immature axons $(\mathrm{J})$. In $\mathrm{J}$, it can be appreciated how immature axons travel in parallel toward the $\mathrm{CA} 3 \mathrm{region}$. $\mathrm{K}-\mathrm{O}$ ) wt aged mice (12 months old) show a decrease in the number of immature neurons (K, M, N) as well as a reduction in the number of immature axons ( $\mathrm{N}, \mathrm{O})$. However, in $\mathrm{O}$, it can be observed how organization of immature axons remains unaltered compared to that of young wt animals (E, J). However, the density of axons is drastically reduced compared to that of young wt animals (E, J). P-T) In a murine model of Frontotemporal Dementia linked to Parkinsonism associated to chromosome 17 (FTDP-17) (TauVLW mice), in which mutated human tau expression is known to be related to a dramatic reduction in the number of immature neuroblasts $(\mathrm{P}, \mathrm{R}, \mathrm{S})$, immature axons appear slightly disorganized in the hilar region and a reduced number of axons can be appreciated in this region (T). U-Y) In glycogen synthase kinase $3 \beta$-overexpressing mice (GSK3 $\beta$-OE mice), the number of immature neuroblasts is known to be drastically increased compared to that of age-matched wt mice, as can be observed in $\mathrm{U}, \mathrm{W}$ and $\mathrm{X}$. X and Y show the increased density of axonal processes that can be appreciated in the hilus of this animal model, correlating with the increase in the number of immature neuroblasts. Z-AD show representative images of an aged A $\beta P P i n d$, sw mouse ( 9 months of age), in which two amyloid- $\beta$ plaques can be observed in the molecular layer and the hilus, respectively (red asterisks) ( $Z$ and AA). As can be seen in $\mathrm{Z}, \mathrm{AB}$, and $\mathrm{AC}$, no immature neuroblasts can be observed in this animal surrounding amyloid- $\beta$ plaques nor can immature axons be detected (AD). GCL, granule cell layer; H: hilus; ML, molecular layer. White triangles: 3R-Tau ${ }^{+}$axons. 
Authors' disclosures available online (http://www.jalz.com/disclosures/view.php?id=1142).

\section{REFERENCES}

[1] Zhao C, Teng EM, Summers RG Jr, Ming GL, Gage FH (2006) Distinct morphological stages of dentate granule neuron maturation in the adult mouse hippocampus. $J$ Neurosci 26, 3-11.

[2] Jones SP, Rahimi O, O’Boyle MP, Diaz DL, Claiborne BJ (2003) Maturation of granule cell dendrites after mossy fiber arrival in hippocampal field CA3. Hippocampus 13, 413427.

[3] Doetsch F, Hen R (2005) Young and excitable: The function of new neurons in the adult mammalian brain. Curr Opin Neurobiol 15, 121-128.

[4] Seri B, Garcia-Verdugo JM, Collado-Morente L, McEwen BS, Alvarez-Buylla A (2004) Cell types, lineage, and architecture of the germinal zone in the adult dentate gyrus. J Comp Neurol 478, 359-378.

[5] Hastings NB, Gould E (1999) Rapid extension of axons into the CA3 region by adult-generated granule cells. J Comp Neurol 413, 146-154.

[6] Toni N, Laplagne DA, Zhao C, Lombardi G, Ribak CE, Gage FH, Schinder AF (2008) Neurons born in the adult dentate gyrus form functional synapses with target cells. Nat Neurosci 11, 901-907.

[7] Ming GL, Song H (2011) Adult neurogenesis in the Mammalian brain: Significant answers and significant questions. Neuron 70, 687-702.

[8] Andreadis A, Brown WM, Kosik KS (1992) Structure and novel exons of the human tau gene. Biochemistry 31, 1062610633.

[9] Goedert M, Spillantini MG, Jakes R, Rutherford D, Crowther RA (1989) Multiple isoforms of human microtubuleassociated protein tau: Sequences and localization in neurofibrillary tangles of Alzheimer's disease. Neuron 3, 519526.

[10] Lovestone S, Reynolds CH (1997) The phosphorylation of tau: A critical stage in neurodevelopment and neurodegenerative processes. Neuroscience 78, 309-324.

[11] Goedert M, Spillantini MG, Hasegawa M, Jakes R, Crowther RA, Klug A (1996) Molecular dissection of the neurofibrillary lesions of Alzheimer's disease. Cold Spring Harb Symp Quant Biol 61, 565-573.

[12] Goedert M, Spillantini MG (2001) Tau gene mutations and neurodegeneration. Biochem Soc Symp, 59-71.

[13] Lee VM, Goedert M, Trojanowski JQ (2001) Neurodegenerative tauopathies. Annu Rev Neurosci 24, 1121-1159.

[14] Avila J, Lucas JJ, Perez M, Hernandez F (2004) Role of tau protein in both physiological and pathological conditions. Physiol Rev 84, 361-384.

[15] Llorens-Martin M, Torres-Aleman I, Trejo JL (2006) Pronounced individual variation in the response to the stimulatory action of exercise on immature hippocampal neurons. Hippocampus 16, 480-490.

[16] Lim F, Hernandez F, Lucas JJ, Gomez-Ramos P, Moran MA, Avila J (2001) FTDP-17 mutations in tau transgenic mice provoke lysosomal abnormalities and Tau filaments in forebrain. Mol Cell Neurosci 18, 702-714.
[17] Lucas JJ, Hernandez F, Gomez-Ramos P, Moran MA, Hen R, Avila J (2001) Decreased nuclear beta-catenin, tau hyperphosphorylation and neurodegeneration in GSK-3beta conditional transgenic mice. EMBO J 20, 27-39.

[18] Hsia AY, Masliah E, McConlogue L, Yu GQ, Tatsuno G, Hu K, Kholodenko D, Malenka RC, Nicoll RA, Mucke L (1999) Plaque-independent disruption of neural circuits in Alzheimer's disease mouse models. Proc Natl Acad Sci U S A 96, 3228-3233.

[19] Bullmann T, de Silva R, Holzer M, Mori H, Arendt T (2007) Expression of embryonic tau protein isoforms persist during adult neurogenesis in the hippocampus. Hippocampus 17, 98102.

[20] Fuster-Matanzo A, de Barreda EG, Dawson HN, Vitek MP, Avila J, Hernandez F (2009) Function of tau protein in adult newborn neurons. FEBS Lett 583, 3063-3068.

[21] Hong XP, Peng CX, Wei W, Tian Q, Liu YH, Yao XQ, Zhang Y, Cao FY, Wang Q, Wang JZ (2010) Essential role of tau phosphorylation in adult hippocampal neurogenesis. Hippocampus 20, 1339-1349.

[22] Lazarov O, Mattson MP, Peterson DA, Pimplikar SW, van Praag H (2010) When neurogenesis encounters aging and disease. Trends Neurosci 33, 569-579.

[23] Llorens-Martin M, Hernandez F, Avila J (2011) Expression of frontotemporal dementia with parkinsonism associated to chromosome 17 tau induces specific degeneration of the ventral dentate gyrus and depressive-like behavior in mice. Neuroscience 196, 215-227.

[24] Sirerol-Piquer M, Gomez-Ramos P, Hernandez F, Perez M, Moran MA, Fuster-Matanzo A, Lucas JJ, Avila J, GarciaVerdugo JM (2011) GSK3beta overexpression induces neuronal death and a depletion of the neurogenic niches in the dentate gyrus. Hippocampus 21, 910-922.

[25] Weingarten MD, Lockwood AH, Hwo SY, Kirschner MW (1975) A protein factor essential for microtubule assembly. Proc Natl Acad Sci U S A 72, 1858-1862.

[26] Drechsel DN, Hyman AA, Cobb MH, Kirschner MW (1992) Modulation of the dynamic instability of tubulin assembly by the microtubule-associated protein tau. Mol Biol Cell 3, 1141-1154.

[27] Goedert M, Jakes R (1990) Expression of separate isoforms of human tau protein: Correlation with the tau pattern in brain and effects on tubulin polymerization. EMBO J 9, 4225-4230.

[28] Kosik KS, Orecchio LD, Bakalis S, Neve RL (1989) Developmentally regulated expression of specific tau sequences. Neuron 2, 1389-1397.

[29] Janke C, Beck M, Stahl T, Holzer M, Brauer K, Bigl V, Arendt T (1999) Phylogenetic diversity of the expression of the microtubule-associated protein tau: Implications for neurodegenerative disorders. Mol Brain Res 68, 119-128.

[30] Kampers T, Pangalos M, Geerts H, Wiech H, Mandelkow E (1999) Assembly of paired helical filaments from mouse tau: Implications for the neurofibrillary pathology in transgenic mouse models for Alzheimer's disease. FEBS Lett 451, 39-44.

[31] Brion JP, Smith C, Couck AM, Gallo JM, Anderton BH (1993) Developmental changes in tau phosphorylation: Fetal tau is transiently phosphorylated in a manner similar to paired helical filament-tau characteristic of Alzheimer's disease. $J$ Neurochem 61, 2071-2080.

[32] Spillantini MG, Goedert M (1998) Tau protein pathology in neurodegenerative diseases. Trends Neurosci 21, 428-433. 